Article

\title{
Analysis of Mass Transport through Anisotropic, Catalytic/Bio-Catalytic Membrane Reactors
}

\author{
Endre Nagy *(D) and Márta Vitai \\ Chemical and Biochemical Procedures Laboratory, Institute of Biomolecular and Chemical Engineering, \\ University of Pannonia, Egyetem u. 10, H-8200 Veszprém, Hungary; vitai.marta@gmail.com \\ * Correspondence: nagy@mukki.richem.hu; Tel.: +36-20-351-8725
}

Received: 1 March 2019; Accepted: 7 April 2019; Published: 13 April 2019

check for updates

\begin{abstract}
This paper investigated the steady-state mass transport process through anisotropic, composite membrane layers with variable mass transport coefficients, such as the diffusion coefficient, convective velocity, or chemical/biochemical reaction rate constant. The transfer processes can be a solution-diffusion model or diffusive plus convective process. In the theoretical part, the concentration distribution as well as the inlet and outlet mass transfer rates' expressions are defined for physical transport processes with variable diffusion or solubility coefficients and then that for transport processes accompanied by first- and zero-order reactions, in the presence of diffusive and convective flow, with constant and variable parameters. The variation of the transport parameters as a function of the local coordinate was defined by linear equations. It was shown that the increasing diffusion coefficient or convective flow induces much lower concentrations across the membrane layer than transport processes, with their decreasing values a function of the space coordinate. Accordingly, this can strongly affect the effect of the concentration dependent chemical/biochemical reaction. The inlet mass transfer rate can also be mostly higher when the transport parameter decreases across the anisotropic membrane layer.
\end{abstract}

Keywords: transport across anisotropic membrane; variable diffusion coefficient; variable convective velocity; first-order reaction; zero-order reaction; inlet mass transfer rate; outlet mass transfer rate

\section{Introduction}

The thermal motion of the transferred components through a dense membrane layer has basically the same transfer laws as that through a laminar liquid or gas phase. The most important mass transfer expressions for gas-liquid or liquid-liquid systems, without [1,2] and with chemical reaction [1,3,4] with constant or variable diffusion coefficients [1], were described in detail in previous literature [1-4]. Though the diffusion process, at least during single component transport, has a similar mechanism in the membrane layer than in a laminar fluid layer, against which there are two essential differences in the mass transport processes between the two cases [5,6], namely: (i) A convective velocity can exist in membrane transport, which can increase or decrease the overall (diffusive plus convective) flux, depending on the membrane's structure, the transmembrane pressure difference, and the interconnection between molecules, etc.; (ii) the absence or presence of a sweeping phase on the permeate side. Due to these conditions, the outlet boundary conditions differ from each other in these two operating modes. The presence of a sweeping phase can induce the same outlet boundary condition that occurs during mass transfer through the laminar fluid phase into its bulk fluid phase. It is important to note that the often strong physico-chemical connection between the simultaneously transported molecules and the polymer molecules can induce special transport mechanisms, e.g., coupling transport of components expressed by the Maxwell-Stefan approach etc. [6-11]. These latest 
transport properties, however, mostly do not play important roles in catalytic/biocatalytic processes, thus they are not the subject of this study.

Membranes can be intrinsically catalytic $[12,13]$ or serve as a porous supporting layer for the catalyst [13-15] and/or biocatalyst particles [16,17] [6] (pp. 381-413). The effect of chemical/biochemical reactions during reactant/substrate transport through a catalytic/biocatalytic membrane layer can strongly depend on the values of the transport parameters, such as the diffusion coefficient and convective velocity, which can be affected by both reactant concentrations (usually linear or exponential concentration dependency of the diffusive flow are assumed $[18,19])$. The convective velocity can vary with the diffusive fluxes, but mostly it is determined by the transmembrane pressure difference, according to the Darcy law [20]. The diffusion coefficient can change as a function of the reactant(s) concentration and, as a function of the space coordinate due to inhomogeneity of the membrane's structure. As an example, the creation of the anisotropic membrane might be the thermally induced phase separation process [21,22] or preparation of the multilayer composite membrane [23,24]. The value of the superficial convective velocity can vary across the membrane, depending on its structure, porosity change, and/or the change of the volumetric flow rate due to a reaction, etc. The effect of the chemical reactions with different reaction kinetics was relatively intensively studied in the literature for simple diffusive transport $[12,13]$, but perhaps less intensively for the diffusive plus convective transport process [25-32].

This study focuses on the reactant(s) transport's description with variable transport parameters (diffusion coefficient, convective velocity) without chemical/biochemical reactions and those accompanied by chemical/biochemical reactions, assuming constant and variable diffusion coefficients and/or reaction rate constant [6], as well as with constant and variable solvent/solute convective velocities. The effect of the variable convective velocity is of emphasized importance in this discussion. The direction of its variation, as a function of the local coordinate, i.e., whether it increases or decreases, can essentially affect the transport process. This transport problem has not been discussed in detail in the literature to our knowledge yet. An analytical approach solution method was developed and used, which reduces the diffusive-convective transport with the reaction into a transport with constant parameters and accompanied by first-order ones, which is already solvable analytically. Both operating modes, namely without and with a sweeping phase on the permeate side, are subjects of this investigation by applying different transport properties.

\section{Diffusive (Plus Convective) Mass Transport through a Flat-Sheet Membrane, without Chemical/Biochemical Reactions}

In this section, the physical mass transfer rate and also the interfacial concentrations between the sublayers are defined in cases of variable diffusion and/or solubility coefficients. The membrane layer is divided into $N$ sublayers with the same thickness, in which both the diffusion coefficient and the solubility coefficients are assumed to be constant. This situation is illustrated in Figure 1, thus the concentration distribution is linear in every sublayer, when there is no chemical reaction and/or convective flow, during transport. It is worth noting that during diffusive transport without a chemical reaction, concentration gradient, in the membrane layer can only be created when there is a sweeping phase on the permeate side. This flowing fluid phase removes the permeated components from the membrane's permeate side surface, creating a concentration gradient on this outlet surface. The operation mode without a sweeping phase does not induce diffusive flow inside the membrane and the membrane outer surface, only convective flow can transport reactant(s) through the membrane layer (thus, the outlet membrane concentration will be equal to each side of the outer surface on the permeate side, when there is no chemical reaction on the membrane layer; on the other hand, the outlet membrane concentration will be equal to that of the bulk permeate concentration in the presence of a chemical reaction as well). 


\subsection{Concentration Distribution with Varying Diffusion Coefficient}

Solution-diffusion mass transport is discussed here with a constant solubility coefficient. It is assumed in this subsection that the diffusion coefficient is variable. Expressing the mass transfer rate by means of the variable diffusion coefficient, $D_{i}$, and dividing the membrane layer into $N$ sublayers (Figure 1) with a constant diffusion coefficient within the sublayers, the overall mass transfer rate can be expressed as the mass transfer rate for the $i$ th sublayer: $J_{i}^{o}=D_{i}\left(\phi_{i-1}^{*}-\phi_{i}^{*}\right)$ :

$$
J_{o v}^{o}=\frac{\phi^{*}-\phi_{\delta}^{*}}{\left(\sum_{i=1}^{i=N} \frac{1}{D_{i}}\right) \Delta y}
$$

The overall mass transfer rate is equal to the mass transfer rate related to any differential sublayer, namely $J_{o v}^{o}=J_{i}^{o}$ with $i=1, \ldots, N$, thus it can be given for the first sublayer as:

$$
J_{1}^{o}=\frac{D_{1}}{\Delta y}\left(\phi^{*}-\phi_{1}^{*}\right)=J_{o v}^{o}
$$

Accordingly, the membrane concentration of the inner edge of the first sublayer is:

$$
\phi_{1}^{*}=\phi^{*}-J_{o v}^{o} \frac{\Delta y}{D_{1}}
$$

Accordingly, any interface concentration can be predicted as:

$$
\phi_{i}^{*}=\phi_{i-1}^{*}-J_{o v}^{o} \frac{\Delta y}{D_{i}} i=2, \ldots, N
$$

By means of the above simple expression, the concentration distribution can be calculated by any variation of the diffusion coefficient as a function of the local coordinate and/or concentration. In this latter case, the trial-error method should be used to fit the value of the diffusion coefficient to the actual values of the interface concentrations in the sublayers.

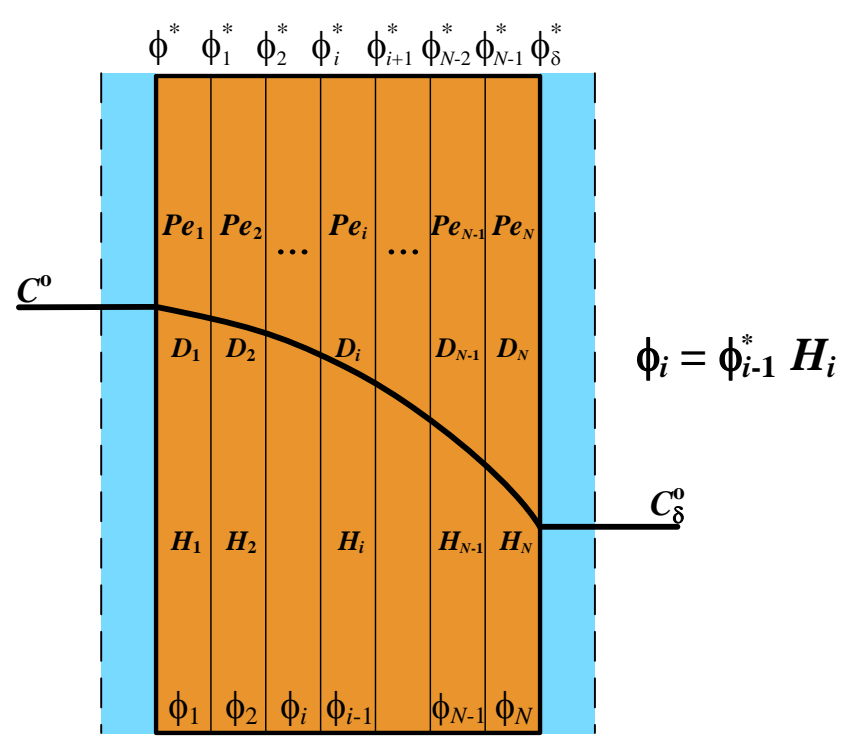

Figure 1. Division of an inhomogeneous (anisotropic) or composite membrane into $N$ thin sublayers, in which the transport parameters, namely the diffusion coefficient $\left(D_{i}\right)$ and Peclet numbers $\left(P e_{i}\right)$, as well as the solubility coefficients $\left(H_{i}\right)$, are constant. 


\subsection{Mass Transport with Varying Diffusion and Solubility Coefficients}

In the case of a multilayer composite membrane, the change of the solubility coefficients should also be taken into account. The notification of the sublayers is illustrated in Figure 1. Every sublayer might have a deviating solubility component. It is assumed that equilibrium exists at the internal interfaces, thus it can be expressed between the two sides of sublayers as: $\phi_{1}=H_{1} \phi^{*}, \phi_{i}=H_{i} \phi_{i-1}^{*}$, $(i=2, \ldots, N) ; \phi_{N}=\phi_{\delta}^{*} ; \phi_{\delta}^{*}=H_{\delta} C_{\delta}^{o}$; (here, values of $\phi_{i}^{*}$ with $i=1, \ldots, N$ refer to the membrane concentration of the outer edge of the $i$ th sublayer, thus, e.g., the mass transfer rate for the $i$ th sublayer can be expressed as:

$$
J_{i}^{o}=\frac{D_{i}}{\Delta y}\left(H_{i-1} \phi_{i-1}^{*}-\phi_{i}^{*}\right)
$$

The overall mass transfer rate can be given for $N$ sublayers as:

$$
J_{i}^{o}=\beta_{o v}^{o}\left(\sum_{i=1}^{N} H_{i} \phi^{*}-\phi_{\delta}^{*}\right)
$$

with:

$$
\frac{1}{\beta_{o v}^{o}}=\left(\sum_{i=1}^{i=N} \frac{1}{D_{i}} \prod_{j=i}^{j=N} H_{j}\right) \Delta y
$$

According to Equations (5)-(7), the concentration of the $i$ th sublayer can be given as:

$$
\phi_{i}^{*}=H_{i-1} \phi_{i-1}^{*}-J_{o v}^{o} \frac{\Delta y}{D_{i}} i=2, \ldots, N
$$

while the value of the $\phi_{1}^{*}$ is:

$$
\phi_{1}^{*}=\phi^{*}-J_{o v}^{o} \frac{\Delta y}{D_{1}}
$$

\subsection{Diffusive Plus Convective Mass Transport with a Variable Peclet Number}

For the sake of completeness and because it might play often important role, especially during biochemical processes, the effect of a variable Peclet number in the presence of convective velocity is discussed in this subsection. The convective velocity mostly increases (it depends on its direction) the substrate (reagent) concentration across the anisotropic membrane layer (as it will be shown later), thus its usage can be useful in the case of concentration dependent reaction rates, e.g., in the case of a first-order reaction. The differential mass balance equation in the dimensionless form [6] (p. 230) to be solved in this case is:

$$
\frac{d^{2} \phi}{d Y^{2}}-P e \frac{d \phi}{d Y}=0
$$

with:

$$
P e=\frac{v \delta}{D} ; Y=\frac{y}{\delta}
$$

The general solution of Equation (10) is:

$$
\phi=T e^{P e Y}+S
$$

The mass transfer rate, taking into account both the diffusive and the convective flows, is [6] (p. 231):

$$
J^{o}=\beta^{o}\left(\phi^{*}-e^{-P e} \phi_{\delta}^{*}\right)
$$

with:

$$
\beta^{o}=k^{o} \frac{P e}{1-e^{-P e}}
$$


where:

$$
k^{o}=\frac{D}{\delta}
$$

The change of the Peclet number can be induced by:

- Variation of the diffusion coefficient; and/or by

- Variation of the convective velocity.

This latter one can be considered as a special case, which can occur during transport through a cone-shaped membrane, e.g., through a cylindrical membrane, where the convective velocity is inversely proportional to the radius. Its importance might be especially significant in a gas phase, non-isomolar, catalytic reaction, e.g., $\mathrm{O}_{2}+2 \mathrm{H}_{2}=2 \mathrm{H}_{2} \mathrm{O}$. Here, the volume of the gas phase can continuously change across the membrane due to the reaction (the effect of the reaction is discussed in Section 3). On the other hand, the transport processes inside the fluid phases in the lumen or shell of a capillary membrane can also create volume change. The superficial convective velocity can also change in the case of variable porosity in the membrane (note the convective velocity in Equation (11) is generally related to the whole cross-section of an operation unit, thus it already involves the effect of the porosity). The value of the $\beta_{i}$ mass transfer coefficient will be different in the above mentioned two cases. This process is also illustrated in Figure 1. Thus, the mass transfer coefficients for the $i$ th sublayer $(i=1, \ldots, N)$ in the above two cases will be, respectively:

$$
\beta_{i}^{o}=\frac{v}{1-e^{-P e_{i}}}
$$

or:

$$
\beta_{i}^{o}=\frac{v_{i}}{1-e^{-P e_{i}}}
$$

Equation (16) corresponds to the variable diffusion coefficient and constant convective velocity, while Equation (17) expresses that the convective velocity is a variable parameter; the diffusion coefficient can be constant or variable, as well.

Note, the solubility coefficient is considered to be constant across the whole membrane layer, and its variation with the local coordinate seems to be less important in this case, thus $H C^{\circ}=\phi^{*}$, $H_{\delta} C_{\delta}^{o}=\phi_{\delta}^{*}$. The change of the driving force depends on values of the $P e_{i}$-number as well (note, the driving force is the concentration difference in the parenthesis, e.g., in Equation (18), which involves an exponential factor as well; thus, its value could essentially be different from that obtained in the case of a simple diffusive flow). According to Equation (13), the mass transfer rate for the $i$ th sublayer can be expressed as (when $i=1$ then $\phi_{i-1}^{*}$ will be equal to the inlet value of the membrane concentration, $\phi^{*}$ ):

$$
J_{i}^{o}=\beta_{i}^{o}\left(\phi_{i-1}^{*}-e^{-P e_{i}} \phi_{i}^{*}\right) i=1, \ldots, N
$$

Applying the equality of the $i$ th mass transfer rates, the overall mass transfer rate can easily be obtained as:

$$
J_{o v}^{o}=\beta_{o v}^{o}\left(\phi^{*}-\exp \left(-\sum_{j=1}^{j=N} P e_{j}\right) \phi_{\delta}^{*}\right)
$$

where:

$$
\frac{1}{\beta_{o v}^{o}}=\frac{1}{\beta_{1}^{o}}+\sum_{j=2}^{j=N}\left\langle\frac{\exp \left(\sum_{l=1}^{j-1}\left(-P e_{l}\right)\right)}{\beta_{j}^{o}}\right\rangle
$$

The concentration of the outer edge of the $i$ th sublayer $\left(i=1, \ldots, N-1\right.$, because at $\left.i=N \phi_{N}^{*}=\phi_{\delta}^{*}\right)$ can be obtained by the following equality: 


$$
\beta_{o v}^{o}\left(\phi^{*}-\exp \left(-\sum_{j=1}^{j=N} P e_{j}\right) \phi_{\delta}^{*}\right)=\beta_{i}^{o}\left(\phi_{i-1}^{*}-e^{-P e_{i}} \phi_{i}^{*}\right)
$$

Thus, the concentration of $\phi_{i}^{*}$ is:

$$
\phi_{i}^{*}=\left\{\phi_{i-1}^{*}-\frac{\beta_{o v}^{o}}{\beta_{i}^{o}}\left(\phi^{*}-\exp \left(-\sum_{j=1}^{j=N} P e_{j}\right) \phi_{\delta}^{*}\right)\right\} e^{P e_{i}} I=1,2, \ldots, N-1
$$

The value of $\phi_{1}^{*}$ is found as:

$$
\phi_{1}^{*}=\left(1-\frac{\beta_{o v}^{o}}{\beta_{1}^{o}}\right) e^{P e_{1}} \phi^{*}+\frac{\beta_{o v}^{o}}{\beta_{1}^{o}} \exp \left(-\sum_{j=1}^{j=N} P e_{j}\right) e^{P e_{1}} \phi_{\delta}^{*}
$$

Equations (22) and (23) enable the user to predict the concentration distribution of the reactant across the catalytic membrane layer, and the mass transfer rate at variable diffusion coefficients and/or at a variable convective velocity.

\section{Diffusive (Plus Convective) Mass Transport with Chemical/Biochemical Reactions through a Flat-Sheet Membrane, Applying the Two Operation Modes}

The chemical/biochemical reaction induces the concentration gradient inside the catalytic membrane layer, independently of the operating modes; thus, the reaction creates diffusive concentration transport across the membrane by both operation modes, namely with a sweeping phase $(d \phi / d y>0$ is valid in almost all cases at the outlet surface of the membrane layer) and without a sweeping phase $(d \phi / d y=0$; this can also take place during biochemical catalytic processes) on the permeate side, though there will be not outlet diffusive flow in this latter case. These two operating modes can essentially give different concentration distributions and mass transfer rates. This paper is focused on the transport process with a variable diffusion coefficient, convective velocity, and/or reaction rate constant. For the sake of completeness, however, we give the mass transfer rate expressions in closed mathematical forms for the cases when the differential mass balance expressions can be solved analytically, namely in cases of mass transport accompanied by first- and zero-order reactions, with constant transport parameters (see Appendix A). These expressions are, at least partly, published already in the literature $[6,28,29,32]$. The general differential mass balance equation to be solved for this reactant transport process across a flat-sheet membrane layer and in a steady-state condition is:

$$
\frac{d}{d y}\left(D[\phi, y] \frac{d \phi}{d y}\right)-v(y) \frac{d \phi}{d y}-Q(\phi, y)=0
$$

where $Q$ expresses the reaction rate with general reaction kinetics. Its dimensionless form of the local coordinate will be $\left(D_{o}\right.$ represents the value of the diffusion coefficient, e.g., in an infinitely diluted solution and/or its value at $y=0$ ):

$$
\frac{d \phi}{d Y}\left(\frac{D(\phi, y)}{D_{o}} \frac{d \phi}{d Y}\right)-P e(\phi, y) \frac{d \phi}{d Y}-\frac{\delta^{2}}{D_{o}} Q(\phi, y)=0
$$

with:

$$
P e=\frac{v(y) \delta}{D_{o}}
$$

Let us adopt it to the Michaelis-Menten kinetics with constant parameters:

$$
\frac{d^{2} \phi}{d Y^{2}}-P e \frac{d \phi}{d Y}-\vartheta^{2} \frac{\phi}{K_{m}+\phi}=0
$$


with:

$$
\vartheta=\sqrt{\frac{v_{\max } \delta^{2}}{D}}
$$

The limiting cases of the Michaelis-Menten bio-reaction kinetics are the first-order (if $K_{m}>>\phi$ ) and zero-order (if $K_{m}<<\phi$ ) reactions. As was mentioned previously, there are two operating modes of membrane bioreactors/reactors, namely without and with a sweeping phase on the permeate side. The inlet and outlet mass transfer rates for these mean four cases and are also listed for the sake of completeness in this study, though some of them are published already [28-32]. The boundary conditions are defined as follows:

$$
\text { at } y=0 \phi=\phi^{*}
$$

The above inlet boundary condition is valid for both operating modes. While at the outlet surface, when the sweeping phase does not exist on the permeate side, the concentration gradient is zero, thus:

$$
\text { at } y=\delta \frac{d \phi}{d y}=0
$$

or when there is a sweeping phase on the permeate side, the concentration gradient will mostly be higher than zero:

$$
\text { at } y=\delta \frac{d \phi}{d y}>0 \text { or } \phi=\phi_{\delta}^{*}
$$

The differential mass balance equations in the case of the first-order reactions were defined with constant mass transfer parameters, namely $P e$ and $\vartheta$, and the mass transfer rates are listed for both the first- and zero-order reactions in the Appendix A.

\subsection{Mass Transport without a Sweep Phase}

In this subsection, the general solutions for first-order reactions are defined. The outlet diffusive mass transfer flux in this case will be zero, thus $d \phi / d y=0$ at $Y=1$. The first-order chemical/biochemical reaction occurs perhaps most often in the technology practice. The differential mass balance equation for this case is as [6] (p. 241):

$$
\frac{d^{2} \phi}{d Y^{2}}-P e \frac{d \phi}{d Y}-\vartheta^{2} \phi=0
$$

or in dimensionless form of the space coordinate:

$$
\frac{d^{2} \phi}{d Y^{2}}-P e \frac{d \phi}{d Y}-\vartheta^{2} \phi=0
$$

where:

$$
P e=\frac{v \delta}{D} ; \vartheta=\sqrt{\frac{k_{1} \delta^{2}}{D}}
$$

The general solution of Equation (32) is known in the literature, so the concentration distribution in the catalytic membrane layer can be given as follows:

$$
\phi=T e^{\lambda Y}+S e^{\tilde{\lambda} Y}
$$

with:

$$
\Theta=\sqrt{\frac{P e^{2}}{4}+\vartheta^{2}} ; \lambda=\frac{P e}{2}-\Theta ; \tilde{\lambda}=\frac{P e}{2}+\Theta
$$

The values of the parameters, $T$ and $S$, should be solved by the application of the boundary conditions given by Equations (28) and (29) [6]. The inlet and the outlet mass transfer rates are given in the Appendix A.1. 


\subsection{Mass Transport with a Sweep Phase}

The sweeping phase removes the permeated reactant/product from the outlet membrane surface into the bulk sweeping phase. Accordingly, the outlet interface and the bulk permeate concentrations can differ from each other. Consequently, diffusive flow can also be induced at the outlet membrane surface, due to the concentration difference at the outlet membrane surface. Thus, the external boundary conditions will be as they are given by Equations (28) and (30). The solution methodology is similar to that applied in the previous case. The inlet and outlet mass transfer rates, for both the first- and zero-order reactions, are given in the Appendix A.2.

\subsection{Mass Transport with Variable Mass Transport Parameters}

The differential mass balance equation to be solved for this case is given by Equation (25), while for the Michaelis-Menten kinetics, as the general biochemical reaction kinetics, it is given by Equation (27a) with constant transport parameters. Obviously, this differential equation has no analytical solution, thus it should be solved by a numerical method or by an analytical approach solution. This latter one was developed by Nagy [6] and used for calculation of the mass transport process. This analytical approach solution's procedure is written in detail, e.g., in [6] (pp. 279-281 and pp. 177-183). The essence of this solution is that the bio-catalytic/catalytic membrane layer is divided into $N$ sublayers with a very thin thickness. The value of $N$ should be relatively high, depending on the slope of the concentration change; in our prediction, the value of $N$ was generally changed as: $N=400-600$ ). The non-linear reaction will then be reduced to a first-order reaction with constant mass transport parameters and it should then be solved analytically for every thin sublayer. Thus, for example, the differential mass balance equation with Michaelis-Menten biochemical reaction kinetics will be defined for the $i$ th sublayer as $(i=1, \ldots, N)$ in the general case, namely when every transport parameter $(D, v$, $\left.v_{\max }, K_{m}\right)$ can vary as a function of the local coordinate and/or even of the concentration of the solute:

$$
D_{i} \frac{d^{2} \phi_{i}}{d y^{2}}-v_{i} \frac{d \phi_{i}}{d y}-k_{i} \phi_{i}=0
$$

with:

$$
k_{i}=v_{m a x, i} \frac{\phi_{i}}{K_{m, i}+\phi_{i-1}}
$$

In the case of an anisotropic or composite membrane layer, both the values of $v_{\max }$ and $K_{m}$ can change as a function of the local coordinate. In the dimensionless local coordinate, Equation (35) will be $(i=1, \ldots, N)$ :

$$
\frac{d^{2} \phi_{i}}{d \Upsilon^{2}}-P e_{i} \frac{d \phi_{i}}{d r}-\vartheta^{2}{ }_{i} \phi_{i}=0
$$

with:

$$
\vartheta_{i}=\sqrt{\frac{\delta^{2} k_{i}}{D_{i}}}
$$

and:

$$
P e_{i}=\frac{v_{i} \delta}{D_{i}}
$$

The general solution of Equation (37) for the $i$ th sublayer can easily be defined by taking into account the solution for constant parameters (see Equation (33)). For determination of the $T_{i}$ and $S_{i}$ parameters, two boundary conditions should be given for every single interface between the sublayers as [6] (p. 280):

$$
\left.\left(-\frac{d \phi_{i}}{d Y}+P e_{i} \phi_{i}\right)\right|_{Y=Y_{i}^{+}}=\left.\left(-\frac{d \phi_{i+1}}{d Y}+P e_{i+1} \phi_{i+1}\right)\right|_{Y=Y_{i+1}^{-}} i=1, \ldots, N-1
$$




$$
\left.\phi_{i}\right|_{Y=Y_{i}^{+}}=\left.\phi_{i+1}\right|_{Y=Y_{i+1}^{-}} \quad i=1, \ldots, N-1
$$

Additionally, the boundary conditions at $Y=0$ and $Y=1$ should also be given, which are given by Equations (28) and (29) or (28) and (30) without and with a sweeping phase, respectively. The value of $\phi_{i-1}$ in Equation (36) is known from its calculated data obtained during the previous calculation step (it is also recommended to apply its average values for the $(i-1)$ th sublayer).

\section{Results and Discussion}

In this section, the predicted results will briefly be discussed, focusing on the effect of the variation range of the mass transfer parameters on the concentration distribution and the mass transfer rates. Especially, the concentration distribution can dramatically vary during the diffusive plus convective mass transport processes. This fact can then significantly affect the effect of the chemical/biochemical reaction rate, which can then often improve the reaction efficiency.

\subsection{Mass Transport without Chemical/Biochemical Reaction}

Two cases of physical mass transfer will briefly be discussed in this section, namely simple diffusive mass transport and diffusive plus convective mass transport. In this latter case, either the diffusion coefficient or the convective velocity is varied as a function of the local coordinate. The developed expressions, given in Section 2, can also be applied when the diffusion coefficient is a function of the reactant concentration. In this case, however, an iteration method should be used for the diffusion coefficient to be accurately fitted to the actual value of the reactant concentration. This section will demonstrate the effect of the variable mass transfer coefficient, namely the diffusion coefficient or convective velocity, by one figure for every case.

\subsubsection{Mass Transport with a Variable Diffusion Coefficient in Diffusive Mass Transport}

Let us first show the typical curves of the concentration distribution during the diffusive, physical mass transfer process with a variable diffusion coefficient. The applied mass transfer rate and concentration equations are listed in Section 2.1. The value of the diffusion coefficient as a function of the local coordinate was changed according to the given expression, namely, in the case of its decreasing value, the applied equation was: $D=D_{o}(1+0.1(N-i))$, while in its increasing case: $D=D_{o}(11-0.1(N-i))$, where $D_{o}=1 \times 10^{-9} \mathrm{~m}^{2} / \mathrm{s}$ and $\delta=1 \times 10^{-4} \mathrm{~m}$. Thus, the value of $D$ was varied between $(11-1) \times 10^{-9} \mathrm{~m}^{2} / \mathrm{s}$ and $(1-11) \times 10^{-9} \mathrm{~m}^{2} / \mathrm{s}$, and its linear variation occurred as a function of the local coordinate, respectively. The inlet mass transfer coefficients were obtained to be $4.21 \times 10^{-5} \mathrm{~m} / \mathrm{s}$ and $4.05 \times 10^{-5} \mathrm{~m} / \mathrm{s}$ with increasing and decreasing diffusion coefficients, respectively. It is obvious that the mass transfer rate strongly depends on the range of the diffusion coefficients' change. As can be seen, the concentration distribution can be strongly affected by the variation of the diffusion coefficient as a function of the local coordinate. It is clearly seen that the decreasing value of the diffusion coefficient induces a concentration distribution with higher concentrations. Higher average values of the reactant concentration can essentially increase the reaction rate if the reaction rate is dependent on the concentration. This occurs most often in practical cases. The zero-order reaction is the only deviation from it. A brief analysis of the effect of the concentration dependency, namely exponential and linear diffusion coefficient, is given in the study of [6] (pp. 97-99).

\subsubsection{The Effect of a Variable Pe-Number on the Concentration Distribution}

The Peclet number involves both the value of the linear convective flow and the diffusive mass transfer coefficient. Usually, the convective velocity is considered to be constant during a transfer process. It is often related to the cross-section of the device investigated; here, to the total membrane surface, which takes into account the average value of the linear velocity. On the other hand, the porosity or tortuosity can change across a membrane with an anisotropic structure as a function of the local coordinate, which can change the superficial convective flow and thus, the average velocity 
related to the total membrane cross-section can also vary across the membrane. The effect of a variable diffusion coefficient with constant convective flow as well as the effect of a variable convective with constant diffusivity will be briefly illustrated. Generally, the effect of the convective velocity can strongly depend on the value of the transmembrane pressure. According to the Darcy law, its value practically linearly increases with the value of the transmembrane pressure difference.

Figure 2 illustrates the concentration distribution with the change of the diffusion coefficient, and due to it the $P e_{i}$ value also varies, though the convective velocity remains constant (Equation (16)). Parameter values are: $D_{o}=1 \times 10^{-9} \mathrm{~m}^{2} / \mathrm{s} ; \delta=5 \times 10^{-4} \mathrm{~m} ; v=1 \times 10^{-5} \mathrm{~m} / \mathrm{s} ; N=30$. Values of $P e_{i}$ were changed between 0.1 and 0.5 in the case of an increasing $P e$-number, $P e_{i}=(0.5+0.07 i) 0.2$, and between 0.45 and 0.1 in the case of its decreasing value, $P e_{i}=(5-0.15 i) 0.1$. The $\beta$ mass transfer coefficient was predicted by Equation (16), in which the convective velocity is constant. According to this expression, the change of this mass transfer coefficient depends on the $\exp \left(-P e_{i}\right)$ value as well. The difference between the two curves is significant. The curve with a decreasing Pe-number is strongly convex, but that with an increasing $P e$-number has an inflexion point; namely, it transforms from a convex curve to a concave one. The decreasing $P e$-number is a consequence of an increasing diffusion coefficient, thus this curve is in harmony with that obtained in the diffusive mass transport process (see Figure 3). Both curves have higher concentration values than those obtained by decreasing values of the diffusion coefficient. After increasing the variation range of the diffusion coefficient, the curves obtained by decreasing the Pe-number (that means increasing the diffusive mass transfer coefficient) tend to quickly reach unity, while they approach the horizontal axis in the case of an increasing $P e$-number.

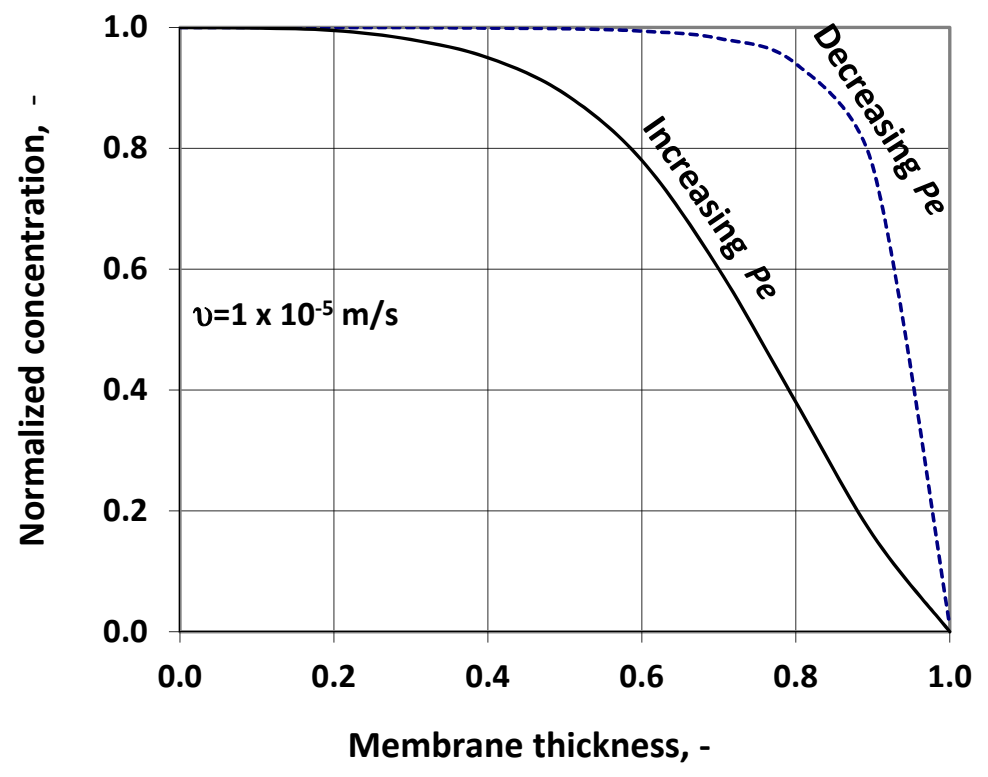

Figure 2. Illustration of the concentration distribution, during diffusive plus convective mass transfer with a variable diffusion coefficient and constant convective velocity, predicted by expressions given in Section 2.3. $\left(D_{o}=1 \times 10^{-9} \mathrm{~m}^{2} / \mathrm{s} ; \delta=5 \times 10^{-4} \mathrm{~m} ; v=1 \times 10^{-5} \mathrm{~m} / \mathrm{s} ; N=30 ; \phi_{\delta}^{*}=0 ; P e_{i}=(0.5+0.07 i) 0.2\right.$ or $P e_{i}=(5-0.15 i) 0.1$. 


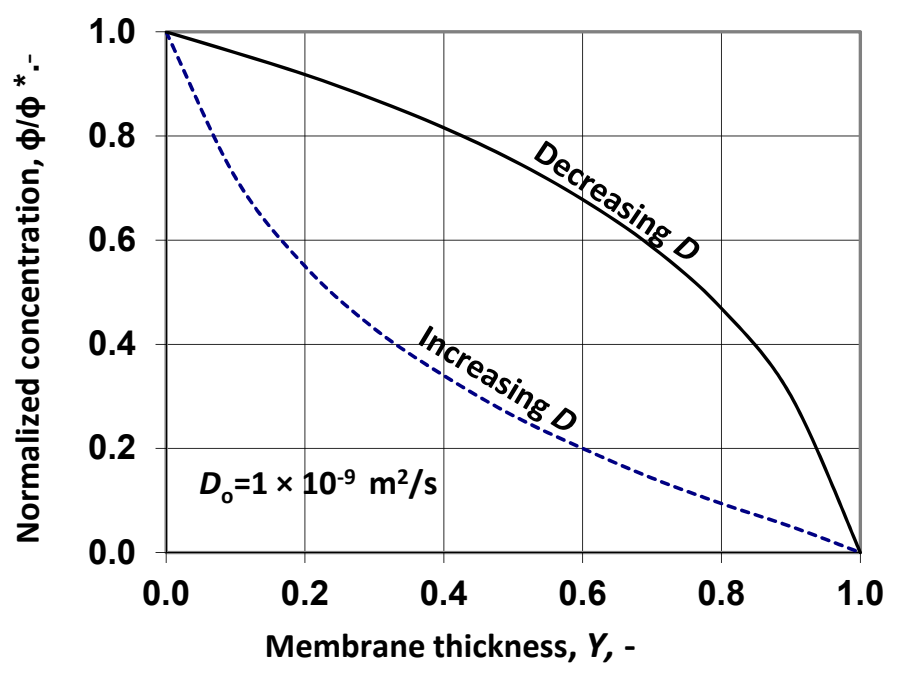

Figure 3. The concentration distribution during diffusive mass transport in the case of a variable increasing $\left(D=D_{o}(11-0.1(N-i))\right.$ or decreasing diffusion coefficient $D=D_{o}(1+0.1(N-i))$ predicted by the expression in Section 2.1; $D_{o}=1 \times 10^{-9} \mathrm{~m}^{2} / \mathrm{s} ; N=100 ; k^{0}=1 \times 10^{-5} \mathrm{~m} / \mathrm{s} ; \delta=1 \times 10^{-4} \mathrm{~m}$; note the membrane thickness means the value of $(Y=y / \delta)$, for all cases plotted).

Let us show briefly the concentration distribution when the diffusion coefficient is constant and the convective velocity changes as a function of the local coordinate. The value of $\beta_{i}$ was calculated by Equation (17). Typical curves are plotted in Figure 4 for illustration of the concentration distribution. Parameters used for calculation were: $D=1 \times 10^{-9} \mathrm{~m}^{2} / \mathrm{s} ; \delta=5 \times 10^{-4} \mathrm{~m} ; k^{o}=0.2 \times 10^{-5} \mathrm{~m} / \mathrm{s} ; v=1$ $\times 10^{-5} \mathrm{~m} / \mathrm{s}, N=50$. The convective velocity was increased according to expressions as: $v_{i}=v(0.2+$ $0.8 i / \mathrm{N})$ or decreased as: $v_{i}=v(1-0.8 i / \mathrm{N})$. Accordingly, the value of $P e_{i}$ was varied between 0.2 and 0.5 or 0.5 and 0.2 to increase or decrease the convective velocity, respectively. The strong increase of the concentration over unity, in the case of lowering the Pe-number, is the result of the decreasing convective velocity. Due to the decreasing convective flow, the slope of the curve gradually increases, causing an increase of the back diffusive flow. After its maximum value, the concentration gradually decreases down to the value of the outlet membrane concentration, namely to zero, since $\phi_{\delta}^{*}=0$ is defined. The diffusive flow should be changed continuously to obtain the constant overall flow, namely the sum of the diffusive plus convective flow. The sum of flows should be constant in every point of the membrane layer. On the other hand, in the case of an increasing convective flow or Peclet number, the concave character of the concentration will slowly become convex close to the outlet membrane surface. Otherwise, the values of the overall mass transfer coefficients, $\beta_{\mathrm{i}}$, are essentially different. Their values are $9.56 \times 10^{-6} \mathrm{~m} / \mathrm{s}$ and $2.97 \times 10^{-6} \mathrm{~m} / \mathrm{s}$ for a decreasing and increasing convective velocity or Peclet number. This can mean that the convective velocity change can essentially alter the inlet overall mass transfer coefficient depending on how its value changes, either increasing or decreasing as a function of the local coordinate.

It might be worthwhile to see how the convective and the diffusive transport rate as well as their sum change as a function of the membrane local coordinate. The question that can obviously arise is what is the reason of the concentration increasing in the case of a decreasing convective velocity? Figure 5 shows the change of the mass transfer rates; that is the diffusive $\left(J^{\circ}\right.$ diff $)$, the convective flows $\left(J^{\circ}\right.$ conv $)$ as well as the sum of these two flows $\left(J^{\circ}{ }^{\circ}\right)$. The overall mass transfer rate (the sum of the diffusive and convective flows) is equal to $9.56 \times 10^{-6} \mathrm{~m} / \mathrm{s}$. As can be seen, it follows from Equation (19) to Equation (21), the overall transfer rate is constant and it is equal to those of any sublayer. Figure 5 represents the overall transfer rate by the red horizontal, dotted line. Meanwhile, the diffusive and the convective flows change as a function of the local coordinate. The increase of the convective flow with the increasing concentration is balanced by the lifting reverse diffusive flow. At a given value of the local coordinate, the diffusive flow will be zero and then its value quickly increases, balancing 
the lowering convective transfer rate. It is perhaps worth noting that the rather low values of the sublayers $(N=50)$ enable the user to gain perhaps a rough prediction of the concentration distribution (the software, written in the Qbasic program language, was not able to calculate higher values of $N$; the accuracy of the calculations was 14 decimal one). These results prove that the plotted values of the concentration are a consequence of the variable convective velocity, and due to it, the variation of the diffusive flow.

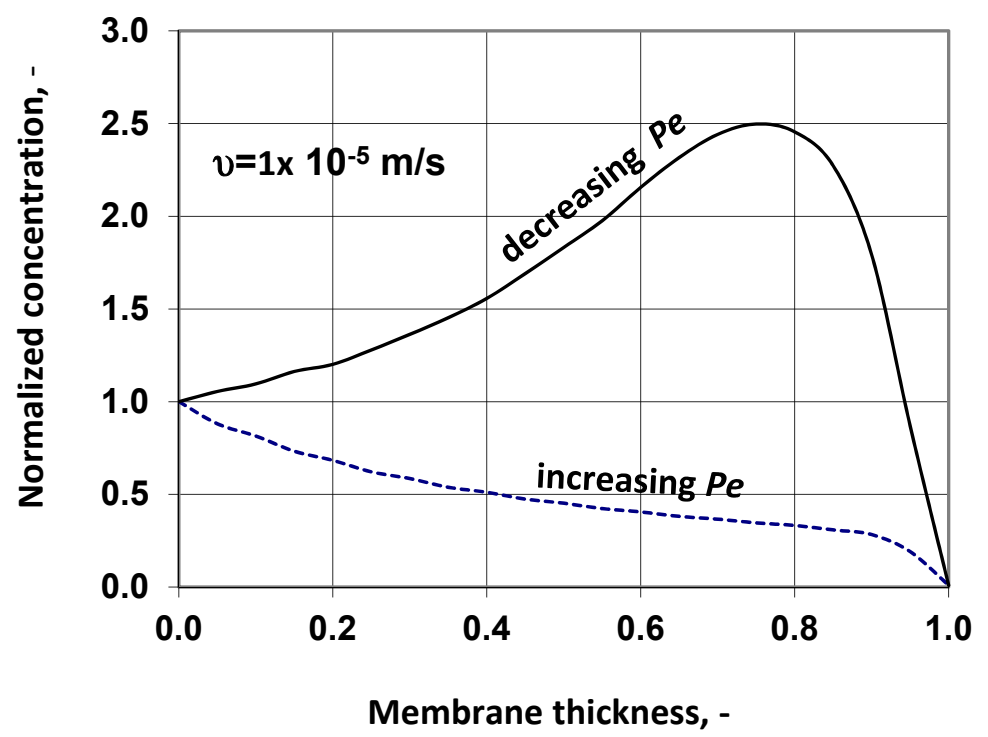

Figure 4. Concentration distribution across the membrane layer with a varying convective velocity and with a constant diffusion coefficient $\left(D=1 \times 10^{-9} \mathrm{~m}^{2} / \mathrm{s} ; \delta=5 \times 10^{-4} \mathrm{~m} ; k^{\mathrm{o}}=0.2 \times 10^{-5} \mathrm{~m} / \mathrm{s}\right.$; $v=1 \times 10^{-5} \mathrm{~m} / \mathrm{s} ; N=50$ ) Values of the convective velocity were changed with its linear function with the space coordinate: Pe-number changed between 0.2 and 0.5 with an increasing, i.e., $v_{i}=v(0.2+$ $0.8 i / N)$, and 0.5 and 0.2 with a decreasing Pe-number with $v_{i}=v(1-0.8 i / N)$.

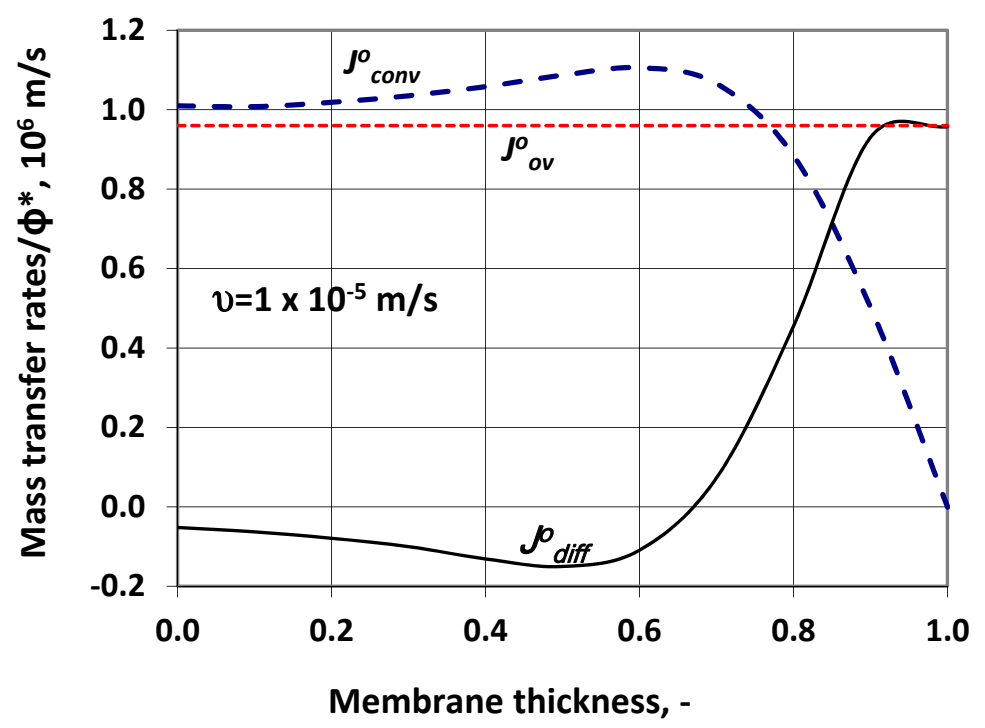

Figure 5. The convective, diffusive, and overall (sum of these two flows) mass transfer rates as a function of the local coordinate predicted by means of the data in Figure 4, with decreasing convective flow (data are the same with those given in the caption of Figure $4\left(v_{i}=v(1-0.8 i / N)\right)$. 


\subsection{Mass Transport with a Chemical Reaction in the Case of Variable Mass Transport Parameters in the Presence of Convective Flow}

In this section, the reactant transfer process accompanied by a chemical/biochemical reaction with constant or variable transport and/or reaction kinetic parameters will briefly be discussed. How the reaction rate can affect the mass transport with constant and variable transfer coefficients will be demonstrated.

\subsubsection{Mass Transport with Constant Transport Coefficients Accompanied by a First-Order Reaction}

The effect of the chemical reaction on mass transport in the presence of convective velocity is rarely discussed in the literature in detail. The importance of this process might particularly be important during biochemical processes [29,31,32]. One of the most important characters for these processes might be the inlet reactant transfer rate, namely the knowledge of its change as a function of the transport and reaction kinetic parameters. The effect of the first-order and also the zero-order reaction has been discussed in books by Nagy [6], in Chapter 8, and by papers of Nagy et al. [6,27-29,32]. Thus, how the concentration distribution varies as a function of the reaction rate is not shown in this subsection, only the change of the mass transfer rate will briefly be discussed. All necessary properties of the transport process can be relatively easily predicted by the equations listed in the appendix, in the cases of first- (Appendices A.1.1 and A.2.1) and zero-order (Appendix A.1.2. and Appendix A.2.2.) reactions. Two figures will illustrate the difference in the mass transfer rates between the two operating modes, namely transport with and without sweeping phases on the permeate side, as a function of the Peclet numbers.

The ratio of the inlet mass transfer rates of the two operating modes, namely that of the transport with and without sweeping phases, is plotted in Figure 6. For an evaluation of the data plotted in this figure, it should be known that the inlet mass transfer rates strongly depend on the reaction rate constant, $\vartheta$. In the case of transport without a sweeping phase, the inlet mass transfer rate tends to be zero when $\vartheta \rightarrow 0$. As was previously mentioned, the concentration gradient cannot be formed in the membrane layer in this case. Accordingly, a small change in the reaction rate can induce a large change in the mass transfer rate during the slow reaction rate regime. This then induces a huge change in the ratio of the mass transfer rates, at low values of $\vartheta$ and low values of convective flow. Note, the convective flux is constant, independently of the reaction rate. On the other hand, these curves illustrate well the effect of the convective velocity, as well, at a given reaction rate constant. With the increase of the $P e$ value, the ratio of the mass transfer rates tends to unity, independently of the values of the reaction rate constant. Similarly, the ratio also approaches unity with the increase of the reaction rate, independently of the value of the convective velocity. Generally, it can be stated that the two operation modes approximately serve the same mass transfer rate as the fast reaction rate, namely when the reaction rate constant is larger than about three, i.e., $\vartheta>$ about 3 in the so-called fast reaction rate regime.

\subsubsection{Mass Transport with Constant Transport Coefficients Accompanied by a Zero-Order Reaction}

Similarly to the results of the first-order reaction, in this case, the mass transfer rates obtained by the two operation modes are also presented. It is worth noting, in the case of the zero-order reaction, that the reaction rate is constant and it is independent of the reactant concentration. Thus, the behaviour of the ratio of the mass transfer rates differs somewhat from that obtained in the case of the first-order reaction. Figure 7 illustrates the effect of the chemical reaction by means of the two operating modes, namely with a sweeping phase (continuous lines) and without a sweeping phase (dotted lines), as a function of the Pe-number. These curves are typical. It tends to a limiting value in the case of transport with a sweeping phase, while it tends to infinite in the case without a sweeping phase, with a decrease of the Pe-number. Obviously, this difference is partly caused by the different behaviour of the values of the physical mass transfer coefficients. Namely, the value of $\beta^{\circ}$ will be zero in the case of only diffusive transport, namely when $P e \rightarrow 0$, and when the transport takes places without a sweeping 
phase, while it remains practically constant in the case of transport with a sweeping phase, during the decreasing Pe-number. On the other hand, with a decrease of the reaction rate modulus, the ratio of the mass transfer coefficients lower to unity in both operating modes. The ratio of the inlet mass transfer coefficient will also tend to unity with an increase of the reaction rate constant (not shown here), similar to the case of the first-order reaction.

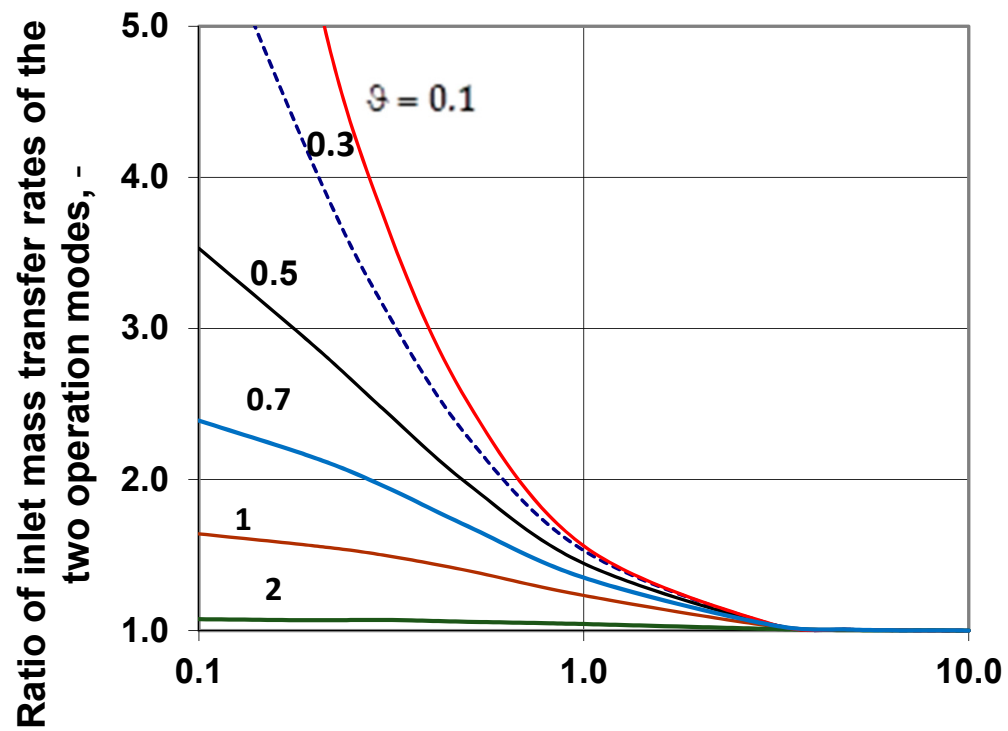

\section{Peclet number, -}

Figure 6. The inlet mass transfer rates, predicted by Equations (A2) and (A14), using the sweeping phase related to that obtained without a sweeping phase, in the case of a first-order reaction, as a function of the $P e$-number by applying constant transport parameters.

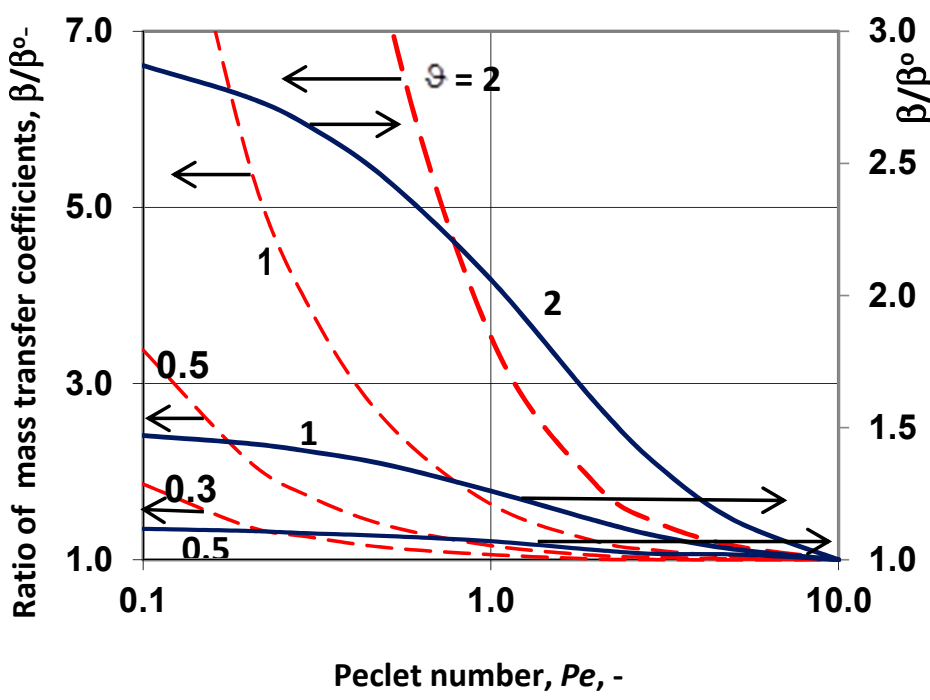

Figure 7. Ratio of the mass transfer coefficients with, $\beta$, and without a chemical reaction, $\beta^{\circ}$, in the cases of both operation modes, namely with a sweeping phase (continuous lines; see Equations (14) and (A21) for $\beta$ and $\beta^{\circ}$ ) and without a sweeping phase (dotted lines; see Equations (14) and (A11) or $\beta$ and $\left.\beta^{\circ}\right)$.

\subsection{Mass Transport with Variable Mass Transport Parameters}

Function of the mass transport parameters (diffusion coefficient, Peclet number, reaction rate constant) can be very different. It can strongly depend on several factors, such as the membrane structure, interconnection between the reactant(s), and membrane molecules, etc. Here, examples will 
be shown to demonstrate the effect of these variable parameters on the transport process. As it was done previously, their closely linear variations as function of the local coordinate are also applied again. For the prediction of the results, the so called analytical approach solution is used for the prediction of the concentration distributions and the mass transfer rates. The expression systems used for calculation are given in detail in [6,30] (pp. 177-183) for transport without convective flow, or in [6] (pp. 312-315) for cases of diffusive plus convective transport, without and with a sweeping phase. As was mentioned previously, all general reaction kinetics can be reduced into a first-order one, where the reaction rate constant involves all other effects using a very thin transfer layer, which enables the user to consider all variable parameters as constant in these sublayers (see Figure 1, which illustrates the division of a membrane layer). The analytical solution should be given for every sublayer (its number was changed during our calculation between 400 and 600, in order to reach the requested accuracy). Thus, using this solution approach enables the reader to apply much higher values of $N$ than were was used in Section 4.1. Obviously, this can then result in a higher accuracy as well. The effect of the reaction is illustrated in this study by two figures, only with a sweeping phase on the permeate side due to its much higher importance in the case of biocatalytic membrane bioreactors. Both figures use a first-order reaction with a constant reaction rate constant, which can be considered as the limiting case of a biochemical reaction rate (see Michaelis-Menten bio-reaction kinetics (Equation (27a)) in the case if $K_{m}>>\phi$. The reaction rate constant in this limiting case is: $k=v_{\max } / K_{m}$ thus, in the case of constant reaction kinetic parameters and diffusion coefficient: $\vartheta=\sqrt{v_{\max } \delta^{2} /\left(K_{m} D\right)}$.

During the calculation, the reaction rate constant and the $P e$ number were varied independently from each other. The Peclet number was varied as a function of the local coordinate according to the expression, $P e_{i}=P e_{0}(1+10 i / N)$ with $N=400$ and $P e_{o}=1$, in Figure 8. Thus, values of the Pecet number were varied between 1 and 11 across the membrane layer. The curves obtained show a similar tendency to those obtained in Section 4.1.2. (Figure 4) without a chemical reaction. Figure 8 clearly shows the effect of the reaction with an increasing Peclet number. Curves with an inflexion point gradually turn to poorly concave ones with the increase of the reaction modulus. The mass transfer rate strongly increases, as can be seen from the concentration gradient's increase, with the increase of the reaction rate at the starting point of the horizontal axis. The concentration distribution with the lowering Peclet number is also interesting.

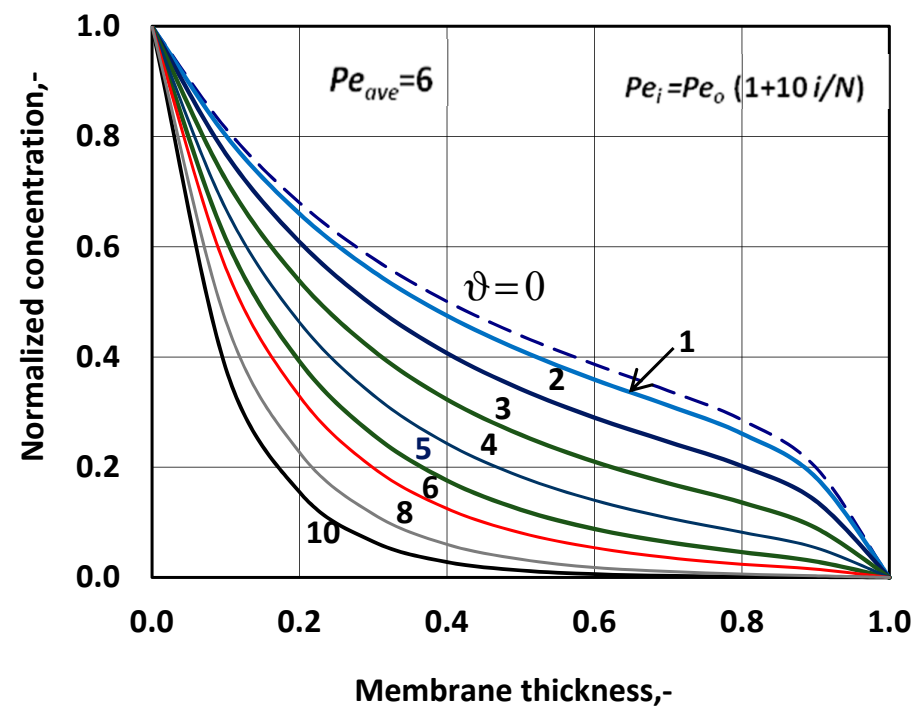

Figure 8. Concentration change with variable increasing Pe number as a function of the local coordinate at different values of the reaction rate constant; the solution of the algebraic equation system is published in [6] (pp. 280-282). ( $D=$ constant; $D=1 \times 10^{-9} \mathrm{~m}^{2} / \mathrm{s} ; N=400 ; P e_{o}=1 ; \phi_{\delta}^{*}=0 P e_{i}=P e_{o}(1+10 i / N)$; $\left.P e_{i}=1-11\right)$. 
Figure 9 illustrates the effect of the decreasing Peclet number on the reactant concentration, at different values of the reaction modulus, namely at $\vartheta=1,2,3,4,5,6,8,10$ with the Peclet number varied by $P e_{i}=P e_{o}(11-10 i / N)$ at values of $P e_{o}=1 ; \phi_{\delta}^{*}=0 ; N=400$. As was experienced previously, the lowering convective velocity increases the reactant concentration as a function of the local coordinate. The increasing concentration also increases the convective flow, thus, its increase will then be balanced by the back diffusion flow, created by the concentration increase. With the decrease of the maximum values of the concentrations due to the increasing reaction rate, the value of the back diffusion flow also gradually decreases. After turning the curves to convex from concave ones, the back diffusion does not exist anymore. Due to the back diffusion, the overall mass transfer rate strongly depends on the range of the Peclet number's change. Thus, generally, it can be stated that the reactant concentration can be higher in the membrane layer in the case of decreasing convective flow, and due to this, the effect of the chemical reaction can be more effective in this case when the concentration depends on the reaction rate. On the other hand, the inlet mass transfer rate can strongly depend on other transport parameters as well. Accordingly, the inlet mass transfer rate and the conversion determines, under given transport parameters, which operating mode is more advantageous. By comparing the concentrations at high values of the reaction modulus, obtained by the two operation modes, it can be seen that the concentration is significantly higher in the case of a decreasing Pe-number, even at $\vartheta=10$. In contrary to that obtained by a constant Peclet number, the two operation modes practically give the same concentration distribution in the fast reaction rate regime $(\vartheta>3-4)[6]$.

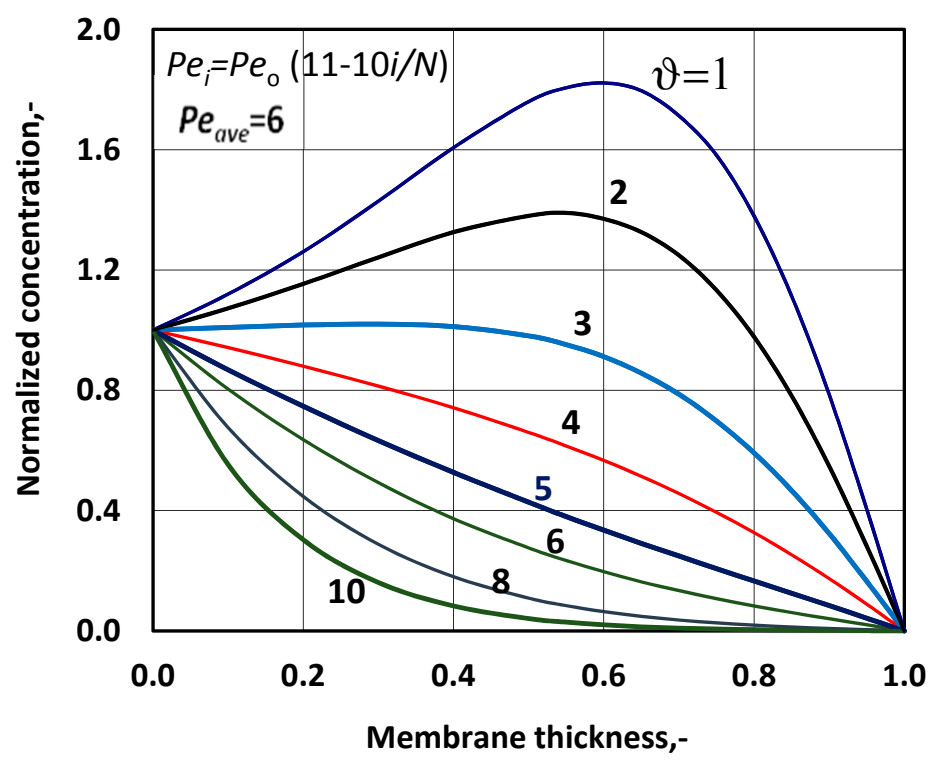

Figure 9. The effect of the chemical reaction in the case of a decreasing Peclet number, as a function of the local coordinate, obtained at different values of the reaction rate $\left(P e_{i}=P e_{0}(11-10 i / N) ; P e_{0}=1\right.$; $\phi_{\delta}^{*}=0 ; N=400 ; D=$ constant).

It is worthwhile to discuss briefly how these significantly different concentration changes and values, due to the variable Peclet number, can affect the inlet mass transfer rate and the conversion in the case of the first-order reaction. The first-order reaction rate linearly depends on the concentration, thus higher concentrations should induce higher reaction rates. The overall inlet mass transfer rates (the sum of the diffusive and convective flows) are illustrated in Figure 10, at three different variations of the $P e$-number, namely with $P e_{i}=P e_{o}(11-10 i / N), P e_{i}=P e_{o}(1+10 i / N)$, and with $P e=$ constant $(P e=6)$. The values of $J / J^{\circ}$ are 3.4, 6.2, and 9.96 at a rather slow chemical reaction modulus, namely at $\vartheta=1$, in the cases of an increasing, constant, and decreasing Peclet number, respectively. The obtained essential differences between the three variation modes are in harmony with the previously shown concentration distribution. The effect of diffusive flow can be significant with the increase of the $P e$-changed range, which increases the concentration gradient on the inlet membrane layer. The inlet 
mass transfer rate, as can be expected, is the highest when the $P e$-number lowers as a function of the space coordinate $\left[P e_{i}=P e_{o}(11-10 i / N)\right]$, accordingly $P e_{a v e}=6$ in the presented case) due to the high inlet Peclet number (it value is 11 at $i=0$ ). Its values are more than three times higher than those obtained by the increasing Peclet number, $P e_{i}=P e_{o}(1+10 i / N)$, again with $P e_{a v e}=6$, but a much lower starting value; $P e=1$ at $i=0$ ). The conversion is also plotted by dotted lines in the three different cases. The conversion's data can essentially differ from each other as a function of the reaction modulus, at any given value of $\vartheta$. The highest values of $X$ were obtained in case of decreasing Peclet number, where the inlet mass transfer rate is also the highest ones. This means that the reacted amount of the solute component can be significantly higher in the case of a decreasing Peclet number due to the essentially higher inlet transfer rate and at higher conversion values. Accordingly, it can be recommended that the membrane structure should be fitted to the requirements of the reaction efficiency, which, in general, should be as high as possible. A more advantageous structure can be predicted by the model equations developed and briefly presented in this study.

\subsection{Complementary Remarks}

Figures 8 and 9 illustrate the effect of the variable convective velocity (the diffusion coefficient was kept constant) on the concentration distribution across the membrane, while Figure 10 plots the effect of the reaction modulus. The variation of the linear velocity can often occur during the gas phase reaction, which can be true to solid-gas biocatalytic processes as well [33] The change of the volumetric/linear velocity can take place not only inside a membrane layer, but in the lumen or shell fluid phases of a capillary membrane or in traditional reactors as well. Let us look at a simple reaction, e.g., the reaction of oxygen with hydrogen into a water molecule:

$$
\mathrm{O}_{2}+2 \mathrm{H}_{2} \Leftrightarrow 2 \mathrm{H}_{2} \mathrm{O}
$$

During this reaction, 3 moles of reactants produce 2 moles of product. The volume change of the gas phase is $2 / 3$ times of the product concentration, assuming that there is no pressure or temperature change. Thus, the differential balance equation across the catalytic membrane can be given for the $i$ th sublayer, taking into account Equation (35), as ( $v_{0}$ means the inlet linear flow rate of the gas phase):

$$
D_{i} \frac{d^{2} \phi_{i}}{d y^{2}}-\frac{2}{3} v_{o} \phi_{i} \frac{d \phi_{i}}{d y}-k_{i} \phi_{i}=0
$$

Equation (43) can easily be solved by the analytical approach method (or numerically) recommended by the authors [6]. Another example could be the carbon dioxide hydrogenation to methanol [34] or its methanation [35]. In the first case, 4 moles of reactants produce 2 moles of product. The volume change can reach $50 \%$. The other important fact during these reactions is that the equilibrium of these reactions strongly depends on the temperature. An application of a membrane process with a suitable metallic membrane is the continuous removal of the product components, which can essentially improve the reactor efficiency. The calculation methodology used here can also be applied for packed bed reactors [36] or photcatalytic reactors when a strong volume change takes place [37]. These additional remarks might help readers to predict transport processes in reactions occurring in a fluid phase in the case of varying transport parameters, though deeper analysis of this topic was not the focus of the present paper.

\section{Conclusions}

Mass transport through an anisotropic or composite membrane can induce variation of the mass transport parameters, which can then strongly affect the mass transfer rate across the membrane layer. This study analysed how the concentration distribution and the mass transfer rates are affected in the presence of variable diffusion coefficients in a solution-diffusion model and variable diffusion coefficients and/or convective velocity in diffusion-convection mass transport. The effect of lowering 
the diffusion coefficient can result in the concentration distribution significantly deviating from that induced by the increasing diffusion coefficient across an anisotropic/composite membrane layer. In the case of a decreasing diffusion coefficient and/or convective velocity, the concentration of the transporting component can be much higher and can even be significantly higher than the inlet concentration than that obtained by increasing the diffusion coefficient or convective velocity. The inlet mass transfer rate can be higher or even lower in the case of a decreasing diffusion coefficient compared to an increasing value. Accordingly, transport processes with different concentrations can have significantly different mass transfer rates. The theoretical prediction of the effect of variable transport parameters enables the user to fit the membrane structure to a given task.

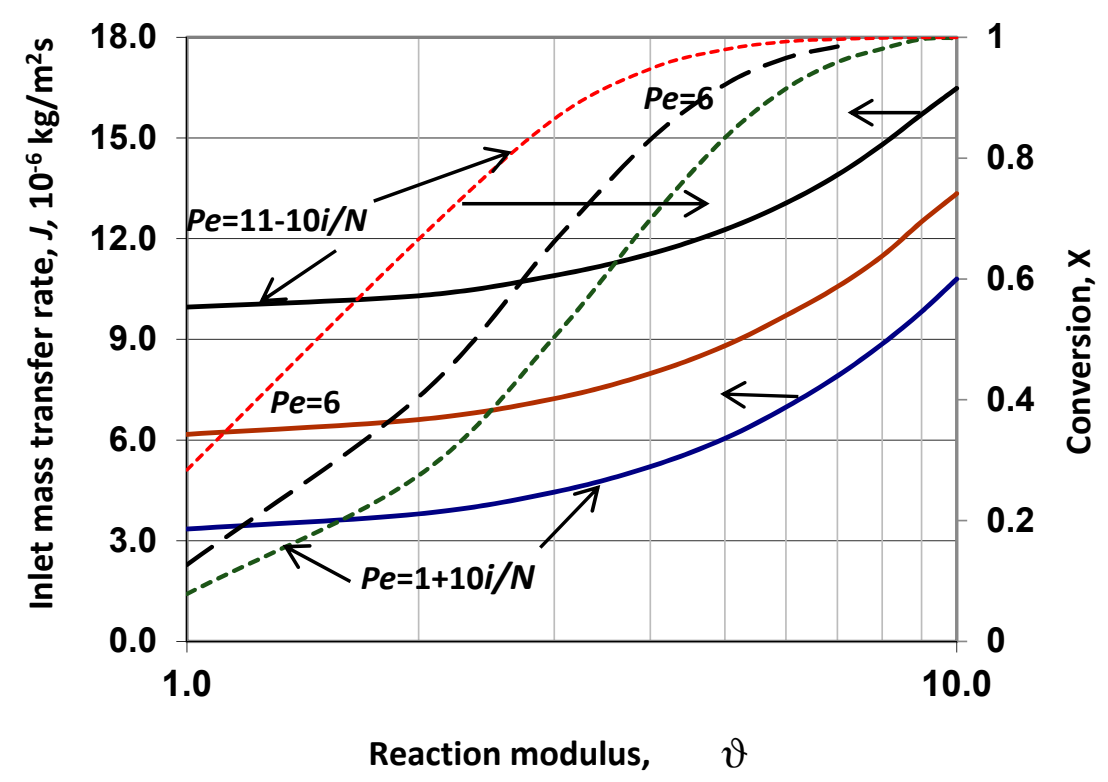

Figure 10. The relative values of the inlet mass transfer rate, $J / J^{\circ}$, and the conversion, $X$, are plotted as a function of the reaction modulus at three different cases, namely with a lowering, increasing, and constant Peclet number, i.e., $P e_{i}=P e_{o}(11-10 i / N) P e_{i}=P e_{o}(1+10 i / N)$ and $P e=$ constant $(P e=6)$, respectively. $\left(P e_{0}=1 ; \phi_{\delta}^{*}=0 ; N=400 ; D=\right.$ constant; $\left.\vartheta_{i}=\sqrt{\delta^{2} k_{i} / D}\right)$.

Author Contributions: Writing—original draft preparation, E.N.; drawing the figures, M.V.

Funding: This research was funded by the National Development Agency grant OTKA, grant number 116727 and GINOP, grant number -2.3.2-15-2016-00017.

Conflicts of Interest: The authors declare no conflict of interest.

\section{Notation}

C fluid solute concentration, $\mathrm{kg} / \mathrm{m}^{3}, \mathrm{~g} / \mathrm{L}$

D diffusion coefficient, $\mathrm{m}^{2} / \mathrm{s}$

$D_{o} \quad$ diffusive mass transfer coefficient at the membrane enters, $\mathrm{m} / \mathrm{s}$

$H \quad$ solubility coefficient

$J^{\circ} \quad$ solute inlet transfer rate, $\mathrm{kg} / \mathrm{m}^{2} \mathrm{~s}$

J inlet mass transfer rate with reaction, $\mathrm{kg} / \mathrm{m}^{2} \mathrm{~s}$

$J_{1} \quad$ inlet mass transfer rate with first-order reaction, $\mathrm{kg} / \mathrm{m}^{2} \mathrm{~s}$

$J_{0} \quad$ inlet mass transfer rate with zero-order reaction, $\mathrm{kg} / \mathrm{m}^{2} \mathrm{~s}$

$k$ reaction rate constant, $\left[k_{1}: 1 / \mathrm{s} ; k_{0}: \mathrm{kg} / \mathrm{m}^{3} \mathrm{~s}\right]$

$k^{o} \quad$ diffusive mass transfer coefficient for the membrane, $\mathrm{m} / \mathrm{s}$

$N \quad$ number of sublayers

Pe membrane Peclet number

$Q \quad$ reaction rate, $\mathrm{kg} / \mathrm{m}^{2} \mathrm{~s}$ 


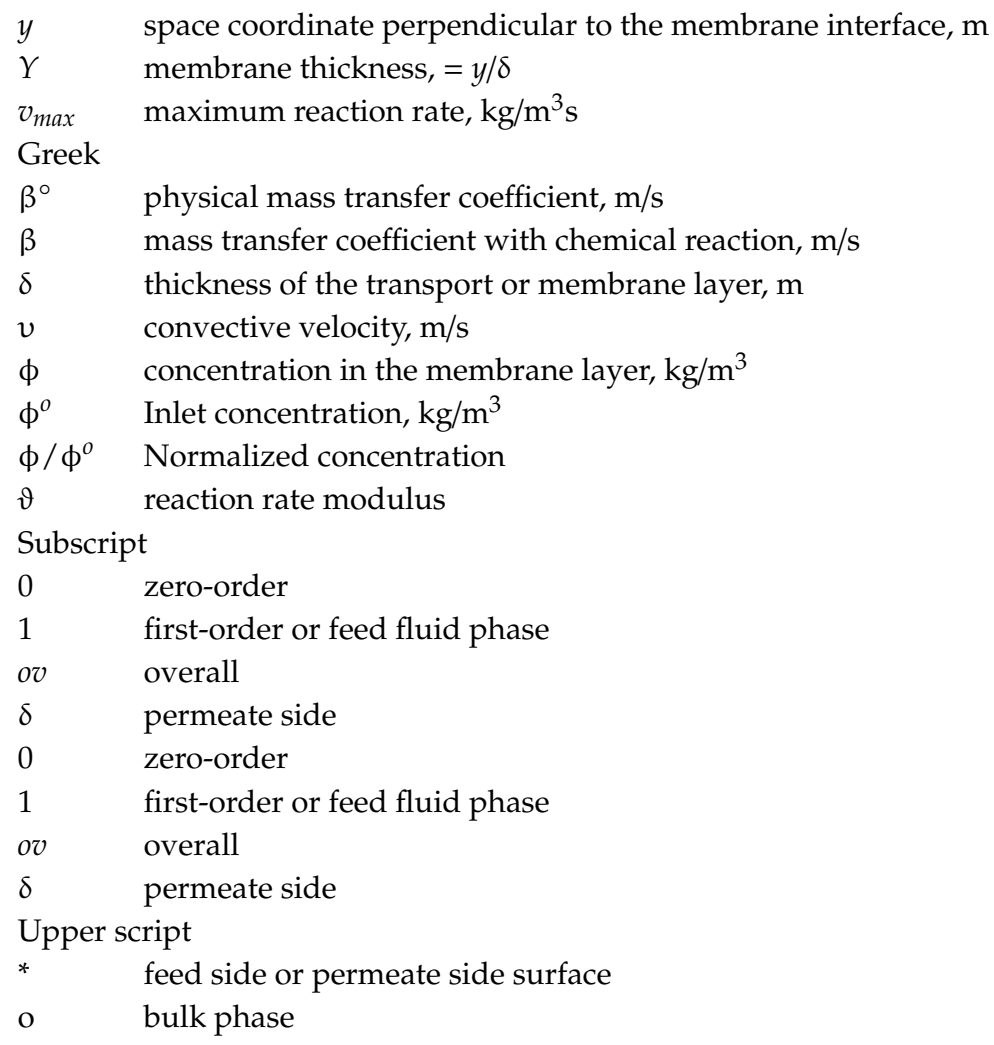

\section{Appendix A}

In this section, the mass transfer rates are obtained by the analytical solution of the mass transport accompanied by first- and zero-order reactions. The two operation modes, for transport without and with a sweep phase on the permeate side, are separately discussed. The role of the sweeping phase is to remove the permeated components from the outer membrane surface. The presence or absence of the sweep phase affects the concentration gradient of the transported component(s) and with it the permeation rate as well.

\section{Appendix A.1. Mass Transport without a Sweep Phase}

In this subsection, the mass transfer rates for first- and zero-order reactions are defined. The outlet diffusive mass transfer flux in this case will be zero, thus $d \phi / d y=0$ at $Y=1$.

\section{Appendix A.1.1. First-Order Chemical/Biochemical Reaction}

The first-order chemical/biochemical reaction perhaps occurs most often in technology practice. The differential mass balance equation for this case is [6] (p. 241):

$$
\frac{d^{2} \phi}{d Y^{2}}-P e \frac{d \phi}{d Y}-\vartheta^{2} \phi=0
$$

The solution of Equation (A1), with the boundary conditions of Equations (28) and (29), gives the inlet $(J)$ and outlet $\left(J_{\delta}\right)$ mass transfer rate as [6] (p. 241):

$$
\begin{gathered}
J=\phi^{*} k^{o} \frac{\left(\frac{P e^{2}}{4}+\Theta^{2}\right) \tanh \Theta+P e \Theta}{\frac{P e}{2} \tanh \Theta+\Theta} \equiv \phi^{*} \beta \\
\beta=k^{o} \frac{\left(\frac{P e^{2}}{4}+\Theta^{2}\right) \tanh \Theta+P e \Theta}{\frac{P e}{2} \tanh \Theta+\Theta}
\end{gathered}
$$

where:

$$
\Theta=\sqrt{\frac{P e^{2}}{4}+\vartheta^{2}}
$$


The outlet mass transfer is:

$$
J_{\delta}=\phi^{*} k^{o} P e \frac{\Theta e^{P e / 2}}{\cosh \Theta\left(\frac{P e}{2} \tanh \Theta+\Theta\right)} \equiv \phi^{*} \beta_{\delta}
$$

where:

$$
\beta_{\delta}=k^{o} P e \frac{\Theta e^{P e / 2}}{\cosh \Theta\left(\frac{P e}{2} \tanh \Theta+\Theta\right)}
$$

The difference between the inlet and the outlet mass transfer rates enables the reader to predict the efficiency of the mass transfer process. Note that a detailed analysis of how the solution can be obtained is given in [6] (pp. 237-249).

\section{Appendix A.1.2. Zero-Order Reaction}

The differential mass balance expression with constant parameters is:

$$
D \frac{d^{2} \phi}{d y^{2}}-v \frac{d \phi}{d y}=k_{0}
$$

By expressing Equation (A7) in the dimensionless form one can get $\left(\Phi=\phi / \phi^{*} ; Y=y / \delta\right)$ :

$$
\frac{d^{2} \Phi}{d Y^{2}}-P e \frac{d \Phi}{d Y}=\vartheta^{2}
$$

where:

$$
\vartheta=\sqrt{\frac{k_{0} \delta^{2}}{D \phi^{*}}}
$$

The inlet mass transfer rate obtained is [6] (p. 264):

$$
\begin{gathered}
J=\beta \phi^{*} \\
\beta=k^{o}\left(\frac{\vartheta^{2}}{P e}\left[1-e^{-P e}\right]+P e\right)
\end{gathered}
$$

The outlet mass transfer rate is:

$$
\begin{gathered}
J_{\delta}=\beta_{\delta} \phi^{*} \\
\beta_{\delta}=k^{0}\left\{\frac{\vartheta^{2}}{P e}+P e-\vartheta^{2}\left(\frac{e^{-P e}}{P e}+1\right)\right\}
\end{gathered}
$$

Appendix A.2. Mass Transport with Sweep Phase

The sweep phase removes the permeated reactant/product from the outlet membrane surface into the bulk sweep phase. Accordingly, the interface and the bulk concentrations can be different from each other. Consequently, diffusive flow can also be induced at the outlet membrane surface, due to the concentration difference at the outlet membrane surface. Thus, the boundary conditions will be as they are given by Equations (28) and (30). This section may also be divided into two subsections, discussing the presence and absence of a sweep phase on the permeate side.

\section{Appendix A.2.1. First-Order Biochemical Reaction}

The inlet mass transfer rate is defined for this case as [6] (p. 245):

$$
\begin{gathered}
J=\beta\left(\phi^{*}-\alpha \phi_{\delta}^{*}\right) \\
\beta=\frac{k^{o}\left(\frac{P e}{2} \tanh \Theta+\Theta\right)}{\tanh \Theta} \\
\alpha=\frac{\Theta e^{-P e / 2}}{\left(\frac{P e}{2}\right) \sinh \Theta+\Theta \cosh \Theta}
\end{gathered}
$$


The outlet mass transfer rate is:

$$
J_{\delta}=\beta_{\delta}\left(\phi^{*}-\alpha_{\delta} \phi_{\delta}^{*}\right)
$$

where:

$$
\beta_{\delta}=\frac{D}{\delta} \frac{\Theta e^{P e / 2}}{\sinh \Theta}
$$

and:

$$
\alpha_{\delta}=\frac{\Theta \cosh \Theta-\frac{P e}{2} \sinh \Theta}{\Theta e^{P e / 2}}
$$

Appendix A.2.2. Zero-Order Biochemical Reaction

The inlet mass transfer rate is obtained as [6] (p. 265):

$$
J=\beta\left(\phi^{*}-\frac{P e^{2}}{\vartheta^{2}-P e^{2} e^{P e}} \phi_{\delta}^{*}\right)
$$

where:

$$
\beta=k^{o}\left(\frac{\vartheta^{2}}{P e}+\frac{\vartheta^{2}-P e e^{P e}}{1-e^{P e}}\right)
$$

The outlet mass transfer rate is:

$$
J_{\delta}=\beta_{\delta}\left(\phi^{*}-\alpha_{\delta} \phi_{\delta}^{*}\right)
$$

with:

$$
\beta_{\delta}=\frac{D}{\delta}\left\{\left(\frac{\vartheta^{2}}{P e}[1-P e]+\frac{\vartheta^{2}-P e e^{P e}}{1-e^{P e}}\right)\right\}
$$

and:

$$
\alpha_{\delta}=\frac{P e}{\beta_{\delta}\left(e^{P e}-1\right)}
$$

\section{References}

1. Crank, J. The Mathematics of Diffusion, 2nd ed.; Clarendon Press: Oxford, UK, 1975; ISBN 0-19-853344-6.

2. Cussler, E.L. Diffusion Mass Transfer in Fluid Systems, 3rd ed.; Cambridge University Press: Cambridge, UK, 2009; ISBN 978-521-87121.

3. Danckwerts, P.V. Gas-Liquid Reaction; McGraw Hill: New York, NY, USA, 1970; ISBN 963-10-1251-4.

4. Westerterp, K.R.; van Swaaij, W.P.M.; Beenackers, A.A.C.M. Chemical Reactor Design and Operation; Wiley and Sons: New York, NY, USA, 1984; ISBN 978-0-471-91730-4.

5. Baker, R.W. Membrane Technology and Application, 2nd ed.; Wiley: Chichester, UK, 2004; pp. 15-84, ISBN 0-470-85445-6.

6. Nagy, E. Basic Equation of Mass Transport through a Membrane Layer, 2nd ed.; Elsevier: Amsterdam, The Netherlands, 2019; ISBN 978-12-813722-2.

7. Heintz, A.; Stephan, W. A generalized solution-diffusion model of the pervaporation process through composite membranes. Part II. Concentration polarization, coupled diffusion and the influence of the porous layer. J. Membr. Sci. 1994, 89, 153-169. [CrossRef]

8. Nagy, E. Nonlinear, coupled mass transfer through a dense membrane layer. Desalination 2004, 163, 345-354. [CrossRef]

9. Izak, P.; Bartovská, L.; Press, K.; Sipek, M.; Uchytill, P. Description of binary liquid mixtures transport through non-porous membrane by modified Maxwell-Stefan equations. J. Membr. Sci. 2003, 214, 293-309. [CrossRef]

10. Flory, P. Principles of Polymer Chemistry; Cornell University Press: New York, NY, USA, 1953; ISBN 978-1-4614-2212-9.

11. Mulder, M. Basic Principles of Membrane Technologies, 2nd ed.; Kluwer Academic Publisher: Amsterdam, The Netherlands, 1996; ISBN 978-94-009-1766-8.

12. Caro, J. Basic Aspect of Membrane Reactors; Elsevier: Amsterdam, The Netherlands, 2010. 
13. Marcano, J.G.S.; Tsotsis, T.T. Catalytic Membranes and Membrane Reactions; Wiley VCH: Veinheim, Germany, 2002; ISBN 9783527302772.

14. Seidel-Morgestern, A. Membrane Reactors; Wiley VCH: Veinheim, Germany, 2010; ISBN 3-527-30979-9.

15. Maria-Grazia, R.-R.; De Angelis, D.; Baschetti, M.G. Models for Facilitated Transport Membranes: A Review. Membranes 2019, 9, 26. [CrossRef]

16. Cabral, J.M.S.; Best, D.; Boross, L.; Tramper, J. Applied Biocatalysis; CFRC Press: New York, NY, USA, 1994; ISBN 9789058230232.

17. Drioli, E.; Giorno, L. Biocatalytic Membrane Reactors; Taylor \& Francis: London, UK, 1999; ISBN 9780748406548.

18. Bitter, J.G.A. Transport Mechanisms in Membrane Separation Processes; Shell Laboratory: Amsterdam, The Netherlands, 1991; ISBN 978-1-4615-3682-6.

19. Schaetzel, P.; Bendjama, Z.; Vaulair, C.; Ngyen, Q.T. Ideal and non-ideal diffusion through polymer: Application to pervaporation. J. Membr. Sci. 2001, 191, 91-102. [CrossRef]

20. Mason, L.A.; Malinaucas, A.P. Gas Transport in Porous Media: The Dusty Gas Model; Elsevier: Amsterdam, The Netherlands, 1983; ISBN 0444421904.

21. Matsuyama, H.; Yuasa, M.; Kitamura, Y.; Teramoto, M.; Lloyd, D.R. Structure control of anisotropic and asymmetric polypropylene membrane prepared by thermally induced phase separation. J. Membr. Sci. 2000, 179, 91-100. [CrossRef]

22. Liang, H.Q.; Wu, Q.Y.; Wan, L.S.; Huang, X.J.; Xu, Z.K. Polar polymer membranes via thermally induced phase separation using a universal crystallizable diluent. J. Membr. Sci. 2013, 446, 482-491. [CrossRef]

23. Li, P.; Wang, Z.; Li, W.; Liu, Y.; Wang, J.; Wang, S. High-Performance Multilayer Composite Membranes with Mussel-Inspired Polydopamine as a Versatile Molecular Bridge for $\mathrm{CO}_{2}$ Separation. Appl. Mater. Interfaces 2015, 7, 15481-15493. [CrossRef]

24. Park, Y.H.; Song, M.A.; Park, C.H.; Nam, S.Y. Fabrication of Multi-Layer Composite Membranes with Excellent $\mathrm{CO}_{2}$ Permeability and Selectivity for Gas Separation Process. J. Nanosci. Nanotechnol. 2017, 17, 7735-7742. [CrossRef]

25. Calabro, V.; Curcio, S.; Iorio, G. A theoretical analysis of transport phenomena in a hollow fiber membrane bioreactor with immobilized biocatalyst. J. Membr. Sci. 2002, 206, 217-241. [CrossRef]

26. Godongawa, B. Effectiveness factor and conversion in a biocatalytic membrane reactor. PLoS ONE 2016, 11, e0153000. [CrossRef]

27. Nagy, E.; Dudas, J.; Mazzei, R.; Giorno, L. Description of the diffusive-convective mass transport in a hollow-fiber biphasic biocatalytic membrane reactor. J. Membr. Sci. 2015, 482, 144-157. [CrossRef]

28. Nagy, E.; Borbély, G. Mass transport through anisotropic membrane layer. Desalination 2009, 240 , 54-63. [CrossRef]

29. Nagy, E.; Kulcsár, E. Mass transport through biocatalytic membrane reactor. Desalination 2009, 245, $422-436$. [CrossRef]

30. Nagy, E. Mass transfer through a convection flow catalytic membrane layer with dispersed nanometer sized catalyst. Ind. Eng. Chem. Res. 2010, 49, 1057-1062. [CrossRef]

31. Nagy, E. Basic equations of mass transfer through biocatalytic membrane layer, Asia-Pac. J. Chem. Eng. 2009, 4, 270-278. [CrossRef]

32. Nagy, E.; Lepossa, A.; Prettl, Z. Mass transfer through a biocatalytic membrane reactor. Ind. Eng. Chem. Res. 2012, 51, 1635-1646. [CrossRef]

33. Kulishova, L.M.; Zharkov, D.O. Solid/Gas Biocatalysis. Biochemistry (Moscow) 2017, 82, 95-105. [CrossRef] [PubMed]

34. Hus, M.; Kopac, D.; Stefancic, N.S.; Jurkovic, D.L.; Dasireddy, D.D.B.C.; Likozar, B. Unravelling the mechanisms of $\mathrm{CO} 2$ hydrogenation to methanol on $\mathrm{Cu}$-based catalysts using first-principles multiscale modelling and experiments. Catal. Sci. Technol. 2017, 7, 5900-5913. [CrossRef]

35. Li, W.; Wang, H.; Jiang, X.; Liu, Z.; Guo, X.; Song, C. A short review of recent advances in $\mathrm{CO}_{2}$ hydrogenation to hydrocarbons over heterogeneous catalysts. RSC Adv. 2018, 8, 7651-7669. [CrossRef] 
36. Pavlisic, A.; Ceglar, R.; Pohar, A.; Likozar, B. Comparison of computational dynamics (CFD) and pressure drop correlations in laminar flow regime for packed bed reactors and columns. Power Technol. 2018, 328, 130-139. [CrossRef]

37. Suhadolnik, L.; Pohar, A.; Novak, U.; Likozar, B.; Mihelic, A.; Ceh, M. Continuous photocatalytic, electrocatalytic and photo-electrocatalytic degradation of a reactive textile dye for wastewater-treatment processes: Batch, microreactor and scaled-up operation. J. Ind. Eng. Chem. 2019, 72, 172-188. [CrossRef]

(c)

(C) 2019 by the authors. Licensee MDPI, Basel, Switzerland. This article is an open access article distributed under the terms and conditions of the Creative Commons Attribution (CC BY) license (http://creativecommons.org/licenses/by/4.0/). 